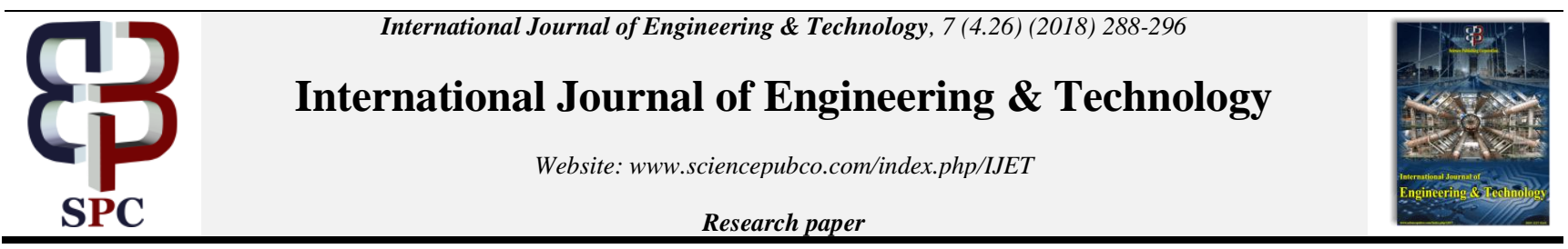

\title{
Branch and Bound Method to Resolve Non-Convex Quadratic Problems Over a Boxed Set
}

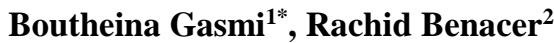 \\ ${ }^{1}$ Laboratoire of (LTM),University Batna 2, Faculty of Maths and informatic 05078Fesdis, Batna, Algeria. \\ ${ }^{2}$ Laboratoire of (LTM),University Batna 2, Faculty of Maths and informatic 05078Fesdis, Batna, Algeria \\ *Corresponding author E-mail: gasmi.boutheina2@gmail.com
}

\begin{abstract}
We present in this paper the technique Branch and Bound with new quadratic approch over a boxed arrangement of Rn. We develop an inexact arched quadratic capacity of the target capacity to decide a lower bound of the worldwide ideal estimation of the first non raised issue (NQP) over every subset of this boxed set. We connected a segment and specialized lessening on the feasable area of (NQP)to quicken the intermingling of the proposed calculation. Finally,we think about the assembly of the proposed calculation and we give a straightforward examination between this strategy and another technique wish have a similar guideline.
\end{abstract}

Keywords: Non Convex Quadratic Programing, Global Optimization, Optimization Methods, Branch and Bound Method, Belinear 0-1 programing.

\section{Introduction}

We consider the accompanying non raised quadratic programming issues:

$$
\left\{\begin{array}{c}
\min f(x)=\frac{1}{2} x^{T} Q x+d^{T} x \\
x \in S \cap\left(D_{f}\right)
\end{array}\right.
$$

where:

$$
\begin{aligned}
S^{0} & =\left\{x \in \mathbb{R}^{n}: L_{i}^{0} \leq x_{i} \leq U_{i}^{0}: i=\overline{1, n}\right\} \\
\left(D_{f}\right) & =\left\{x \in \mathbb{R}^{n}: A x \leq b ; x \geq 0\right\} \\
Q & : \quad \text { is a real }(n \times n) \text { non positive symetric matrix } \\
A & : \quad \text { is a real }(n \times n) \text { symetric matrix } \\
d T & =\left(d_{1}, d_{2}, \ldots, d_{n}\right) \in \mathrm{R}^{n}
\end{aligned}
$$

In ower life ,each thing, each issue is make as a mathematic issues [5], we can likewise

take the statements of Gualili "The word is made at numerical language or scientific issues", specialy "quadratic one".

In this paper we present another square shape Branch and Headed methodology for taking care of non arched quadratic programming issues were we consruct a lower rough raised quadratic elements of the target quadratic capacity f over a boxed arrangement of $\mathrm{Rn}$ introduced as a $\mathrm{n}$-rectangle [2].

This lower inexact capacity is given to decide a lower bound of the worldwide ideal estimation of the first issue (NQP) over every square shape.

The paper is sorted out as followes:
Area 1: In this segment we give a basic presentation of our examinations ; in which we give and characterize the standard type of our concern.

Area 2: another proportional type of the target work proposed as un lower rough straight elements of the quadratic structure over the $\mathrm{n}$ - square shape [6]. We can likewise proposed as an upper inexact direct capacities, however we should regard the procedur of ascertain the lower and the upper bound of the first central square shape $S^{0}$ which noted by $S^{k}=\left[L^{k}, U^{k}\right] \subseteq \mathbb{R}^{n}$ in the $k$-step [4].

Area 3: In this segment we characterize another lower surmised quadratic elements of the quadratic non raised capacity over a nsquare shape as for a square shape to ascertain a lower bound on the worldwide ideal estimation of the first no arched issue (NQP) [7].

Segment 4: We give another straightforward square shape apportioning strategy and depict square shape lessening strategies [3]. Segment 5: Gives another Branch and Decrease Calculation so as to take care of the first non raised issue (NQP).

Segment 6: We examine the intermingling of the proposed Calculation and we give a straightforward examination between this technique and different strategies which have a similar rule [1].

At long last ,a finish of the paper is given to appear and explaine the proficiency of the proposed Calculation.

\section{The Equivalent forms of $f$ over the $n$-rectangle}

In this area we build and characterize the equal type of the non raised quadratic capacity which proposed as a lower inexact straight capacities over an $n$-rectangle $S^{k}=$ $\left[L^{k}, U^{k}\right]$ 
.this work is proposed to decide the lower bound of the worldwide ideal estimation of the first issue (NQP).

Let $\lambda \min$ and $\lambda \max$ be the min eigenvalue and the maximum eigenvalue of the framework $Q$ respectivelly, and we demonstrate the number $\theta$ that $\theta \geq\left|\lambda_{\min }\right|$.

The equivalent linear form of the objective function $f$ is given by:

$$
\begin{aligned}
f(x)= & \left(x-U^{K}\right)^{T}(Q+\theta I)\left(x-U^{K}\right)+d^{T} x-\theta \sum_{i=1}^{n} x_{i}^{2} \\
& +2\left(U^{K}\right)^{T}(Q+\theta I) x-\left(U^{K}\right)^{T}(Q+\theta I) U^{K}
\end{aligned}
$$

by the use of the lower bound $L^{k}$, and is given by:

$$
\begin{aligned}
f(x)= & \left(x-U^{K}\right)^{T}(Q+\theta I)\left(x-U^{K}\right)+d^{T} x-\theta \sum_{i=1}^{n} x_{i}^{2} \\
& +2\left(U^{K}\right)^{T}(Q+\theta I) x-\left(U^{K}\right)^{T}(Q+\theta I) U^{K}
\end{aligned}
$$

by the use of the upper bound $U^{k}$ of the rectangle $S^{k}$. In the other hand, we have the following definition:

Definition 1: Let the function $f: C \subseteq \mathrm{R}^{n} \rightarrow \mathrm{R}$ and $S^{\circ} \subseteq C \subseteq \mathrm{R}^{n} a$ rectangle, the convex envelope of the function $f$ is given by:

$$
\begin{aligned}
& \overline{f_{i}\left(x_{i}\right)}=\delta_{i} x_{i}+\eta_{i}: i=1, n \\
& \text { with: } \\
& \delta_{i}=\frac{f_{i}\left(U_{i}^{\circ}\right)-f_{i}\left(L_{i}^{\circ}\right)}{U_{i}^{\circ}-L_{i}^{\circ}}: i=\overline{1, n} \\
& \eta_{i}=f_{i}\left(L_{i}^{\circ}\right)-\delta_{i} L_{i}^{\circ}: i=\overline{1, n}
\end{aligned}
$$

So, by the use of this definition the convex envelope of the function $h(x)=\left(-x^{2}\right)$ over the interval $S_{j}^{k}=\left[L_{j}^{k}, U_{j}^{k}\right]$ is given by the function:

$$
\bar{h}(x)=-(U j k+L k j) x i+L k j U j k
$$

$n$

which is a linear function, then we get the best linear lower bound of $h(x)={ }^{\mathrm{P}}\left(-x^{2}{ }_{j}\right)$ given

$j=1$

by:

$$
\begin{aligned}
& \phi s k(x) \quad \begin{array}{l}
n \\
\left(-\left(U_{j}+L_{j}\right) x_{i}+L_{j} U_{j}\right) \\
j=1 \\
=
\end{array} \\
&=-\left(U^{k}+L^{k}\right)^{T} x+\left(L^{k}\right)^{T} U^{k}
\end{aligned}
$$

\section{Lower Approximate functions and Error Calculation}

By definition, the initial rectangle $S^{0}$ is given by:

$$
S^{0}=\left\{x \in \mathbb{R}^{n}: L_{i}^{0} \leq x_{i} \leq U_{i}^{0}: i=\overline{1, n}\right\}
$$

We subdivise this rectangle into two sub-rectangles defind by:

$S_{+1}=\left\{x \in \mathbb{R}^{n}: L_{s}^{0} \leq x_{s} \leq h_{s}^{0}: L_{j}^{0} \leq x_{j} \leq U_{j}^{0}: j=\overline{1, n}: j \neq s\right\}$

$S_{+2}=\left\{x \in \mathbb{R}^{n}: h_{s}^{0} \leq x_{s} \leq U_{s}^{0}: L_{j}^{0} \leq x_{j} \leq U_{j}^{0}: j=\overline{1, n}: j \neq s\right\}$

Where, we calculate the point $h_{s}$ by a normal rectangular subdivision ( $\omega$-subdivision).

\subsection{The lower approximate linear function of $f$ over the rectangle $S^{K}$ :}

The best lower inexact direct capacity of the target non raised capacity f over the square shape SK is given in the accompanying hypothesis:

Theorem1 : Let the function $f: C \subseteq \mathrm{R}^{n} \rightarrow \mathrm{R}$ and the rectangle $S^{0}$ $\subseteq \mathrm{R}^{n}$ where $C \subseteq S^{0} \subseteq \mathrm{R}^{n}$, the lower approximate linear function of $f$ is given by:

$L_{S^{K}}(x)=\left(a_{S^{K}}\right)^{T} x+b_{S^{K}}$

$U_{S^{K}}(x)=\left(\overline{a_{S^{K}}}\right)^{T} x+\overline{b_{S^{K}}}$

where:

$a_{S^{K}}=d+2(Q+\theta I) L^{K}-\theta\left(L^{K}+U^{K}\right.$

$b_{S^{K}}=-\left(L^{K}\right)^{T}(Q+\theta I) L^{K}+\theta\left(L^{K}\right)^{T}\left(U^{K}\right)$

$\overline{a_{S^{K}}}=d+2(Q+\theta I) U^{K}-\theta\left(L^{K}+U^{K}\right)$

$\overline{b_{S^{K}}}=-\left(U^{K}\right)^{T}(Q+\theta I) U^{K}+\theta\left(L^{K}\right)^{T}\left(U^{K}\right)_{(8)}$

3.2. The new lower approximate quadratic convex function of $f$ over the rectangle $S^{K}$ :

We utilize the former lower estimated direct capacity of $f$ over the square shape SK to characterize the new lower inexact quadratic arched capacity of $f$ over a similar square shape by:

Definition 2:

$L_{\text {quad }}(x):=L_{S^{K}}(x)-\frac{1}{2} K\left(U^{K}-x\right)\left(x-L^{K}\right)$

$U_{\text {quad }}(x):=U_{S^{K}}(x)-\frac{1}{2} K\left(U^{K}-x\right)\left(x-L^{K}\right)$

and:

where:

$K$ is a positive real number given by the spectral radius of the matrix $(Q+\theta I)$

$$
\theta \quad \geq\left|\lambda_{\min }\right|
$$

$L_{S} K(x)$ the best lower approximate lineair function of $f$ over the rectangle $S^{K}$

\subsection{The New Lower Approximate Linear Function of $f$ over the Rectangle $S^{K}$ :}

By the utilization of the first new lower inexact quadratic capacity of $f$ over the square shape SK we can characterize the new lower estimated straight capacity of $\mathrm{f}$ over a similar square shape by:

\section{Definition 3:}

and:

$\widetilde{L}_{\text {quad }}(x):=L_{S^{K}}(x)-\frac{1}{8} K h^{2}$

$\widetilde{U}_{\text {quad }}(x):=U_{S^{K}}(x)-\frac{1}{8} K h^{2}$

$h:=\left\|U^{\text {with: }}-L^{K}\right\|$

3.3.1. The relation between the convex quadratic approximation and the linear one

We have the following theorem: 
Theorem 3.4 : The tow following inequality are satisfied:

$\widetilde{L}_{\text {quad }}(x):=L_{S^{K}}(x)-\frac{1}{8} K h^{2} \leq L_{\text {quad }}(x) \leq f(x(9)$

$\widetilde{U}_{\text {quad }}(x):=U_{S^{K}}(x)-\frac{1}{8} K h^{2} \leq U_{\text {quad }}(x) \leq f(x$

for all $x \in\left(D_{f}\right) \cap S^{K}$ and $h:=\left\|U^{K}-L^{K}\right\|$ and $\left\|\frac{\partial^{2} f(x)}{\partial x^{2}}\right\| \leq K$

(the normality condition).

Proof: Let the function $g_{1}: \mathrm{R}^{n} \rightarrow \longrightarrow \mathrm{R}$ defind by:

$g_{1}(x)=\widetilde{L}_{\text {quad }}(x)-L_{\text {quad }}(x)$

$=L_{S^{K}}(x)-\frac{1}{8} K h^{2}-\left(L_{S^{K}}(x)-\frac{1}{2} K\left(U^{K}-x\right)\left(x-L^{K}\right)\right)$

$=\frac{1}{2} K\left(-x^{2}+\left(L^{K}+U^{K}\right) x-L^{K} U^{K}-\frac{1}{4}\left\|U^{K}-L^{K}\right\|^{2}\right)$

Passing to the first derivation of $g_{1}$,then, we get:

$\frac{\partial g_{1}}{\partial x}(x)=\frac{1}{2} K\left(-2 x+\left(L^{K}+U^{K}\right)\right)$

Thus:

$\left(\frac{\partial g_{1}}{\partial x}(x)=0\right) \Longleftrightarrow\left(x=\frac{\left(L^{K}+U^{K}\right)}{2}\right)$

The critical point of the function $g_{1}$ is the middle point of the edge $\left[L^{K}, U^{K}\right]$ diatelly, it atteind here max at the middle point

$x^{*}=\frac{(L+U)}{2}$ of $\left[L^{K}, U^{K}\right]_{K}$

$K$, then we have:

$g_{1}(x) \leq \max \left\{g_{1}(x): x \in\left(D_{f}\right) \cap S^{K}\right\}=g_{1}\left(x^{*}\right)=0$

Then;

$L_{\mathrm{e}}$ quad $(x)-L_{q u a d}(x) \leq 0$

In the other hand, we define the function $g_{2}: \mathrm{R}^{n} \rightarrow \mathrm{R}$ given by:

$g_{2}(x)=f(x)-L_{\text {quad }}(x)$

$$
=L_{\text {quad }}(x)-\left(L_{S^{K}}(x)-\frac{1}{2} K\left(U^{K}-x\right)\left(x-L^{K}\right)\right)
$$

Passing to the first derivation of $g_{2}$, then, we get:

$$
\begin{aligned}
\frac{\partial g_{2}}{\partial x}(x) & =\frac{\partial f}{\partial x}(x)-\frac{\partial L_{S^{K}}}{\partial x}(x)+\frac{1}{2} K \frac{\partial}{\partial x}\left(\left(U^{K}-x\right)\left(x-L^{K}\right)\right) \\
& =\frac{\partial f}{\partial x}(x)-a_{S^{K}}+\frac{1}{2} K \frac{\partial}{\partial x}\left(-x^{2}+\left(U^{K}+L^{K}\right) x-L^{K} U^{K}\right. \\
& =\frac{\partial f}{\partial x}(x)-a_{S^{K}}+\frac{1}{2} K\left(-2 x+\left(U^{K}+L^{K}\right)\right)
\end{aligned}
$$

Then, passing to the second derivation:

$\frac{\partial^{2} g_{2}}{\partial x^{2}}(x)=\frac{\partial^{2} f}{\partial x^{2}}(x)-K$

We have the condition:

$\frac{\partial^{2} f(x)}{\partial x^{2}} \leq K$

Then, we obtain:

$\frac{\partial^{2} g_{2}}{\partial x^{2}}(x) \leq 0$

Thus, the function $g_{2}$ is concave over $S^{K}$, and by this we have:

$g_{2}(x) \geq \min \left\{g_{2}(x): x \in S^{K}\right\}=\min \left\{g_{2}\left(L^{K}\right), g_{2}\left(U^{K}\right)\right\}=0$

Then:

$\left(g_{2}(x)=f(x)-L_{q u a d}(x) \geq 0\right)=\Rightarrow L_{q u a d}(x) \leq f(x)$

Finally, we get:

$L_{\mathrm{e}}$ quad $(x) \leq L_{\text {quad }}(x) \leq f(x): x \in S^{K}$

The same thing whene we use the upper bound $U_{\text {quad }}(x)$ with the equivalent linear form of the objective function $f$ and we obtain: $\widetilde{U}_{\text {quad }}(x) \leq U_{\text {quad }}(x) \leq f(x): x \in S^{K}$

\subsection{Approximation errors}

We can assess the guess mistake by the separation between the non arched target work $\mathrm{f}$ and here lower aproximation capacities.

\subsubsection{The linear approximation error}

Is introduced by the separation between the capacity $\mathrm{f}$ and here new lower surmised direct capacity Lequad over the boxed set SK , then we have the accompanying suggestion:

Proposition 3.5 : Let the function $f: C \subseteq \mathrm{R}^{n} \rightarrow \rightarrow \mathrm{R}$ where $C \subseteq S^{0}$ $\subseteq \mathrm{R}^{n}$ and $\theta \geq\left|\lambda_{\min }\right|$ for this the matrix $(Q+\theta I)$ be semi-positive, then we have:

$\max _{x \in S^{K} \cap\left(D_{f}\right)}\left\{\left|f(x)-\widetilde{L}_{\text {quad }}(x)\right|\right\} \leq\left(\rho(Q+\theta I)+\theta+\frac{1}{8} K\right)\left\|U^{K}-L^{K}\right\|^{2}$
$\max _{x \in S^{K} \cap\left(D_{f}\right)}\left\{\left|f(x)-\widetilde{U}_{\text {quad }}(x)\right|\right\} \leq\left(\rho(Q+\theta I)+\theta+\frac{1}{8} K\right)\left\|U^{K}-L^{K}\right\|^{2}$

Proof: we have:

$f(x)-\widetilde{L}_{\text {quad }}(x)=\left(x-L^{K}\right)^{T}(Q+\theta I)\left(x-L^{K}\right)+d^{T} x-\theta \sum_{i=1}^{n} x_{i}^{2}$

$$
\begin{aligned}
& +2\left(L^{K}\right)^{T}(Q+\theta I) x-\left(L^{K}\right)^{T}(Q+\theta I) L^{K}-\left(L_{S^{K}}(x)-\frac{1}{8} K h^{2}\right) \\
= & \left(x-L^{K}\right)^{T}(Q+\theta I)\left(x-L^{K}\right)+d^{T} x-\theta \sum_{i=1}^{n} x_{i}^{2} \\
& +2\left(L^{K}\right)^{T}(Q+\theta I) x-\left(L^{K}\right)^{T}(Q+\theta I) L^{K} \\
& -\left(\left(d+2(Q+\theta I) L^{K}-\left(L^{K}+U^{K}\right)\right)^{T} x+\left(-\left(L^{K}\right)^{T}(Q+\theta I) L^{K}+\left(L^{K}\right)^{T} U^{K}\right)\right) \\
& +\frac{1}{8} K h^{2} \\
= & \left(x-L^{K}\right)^{T}(Q+\theta I)\left(x-L^{K}\right)+\frac{1}{8} K h^{2}+\theta\left(\left(L^{K}+U^{K}\right)^{T} x-x^{T} x-\left(L^{K}\right)^{T} U^{K}\right)
\end{aligned}
$$

In the other hand, we have:

$\left(x-L^{K}\right)\left(U^{K}-x\right)=\left(L^{K}+U^{K}\right)^{T} x-x^{T} x-\left(L^{K}\right)^{T} U^{K}$

Then we get:

$f(x)-\widetilde{L}_{\text {quad }}(x)=\left(x-L^{K}\right)^{T}(Q+\theta I)\left(x-L^{K}\right)+\frac{1}{8} K h^{2}+\theta\left(x-L^{K}\right)\left(U^{K}-x\right)$

so:

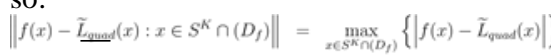

$$
\begin{aligned}
& =\left\|\left(x-L^{K}\right)^{T}(Q+\theta I)\left(x-L^{K}\right)+\frac{1}{8} K h^{2}+\theta\left(x-L^{K}\right)\left(U^{K}-x\right)\right\| \\
& \leq\left\|\left(x-L^{K}\right)^{T}(Q+\theta I)\left(x-L^{K}\right)\right\|+\theta\left\|\left(x-L^{K}\right)\left(U^{K}-x\right)\right\|+\frac{1}{8} I \\
& \leq\left(\rho(Q+\theta I)\left\|U^{K}-L^{K}\right\|^{2}\right)+\theta\left\|U^{K}-L^{K}\right\|^{2}+\frac{1}{8} K h^{2} \\
& \leq\left(\rho(Q+\theta I)+\theta+\frac{1}{8} K\right) h^{2}: h^{2}=\left\|U^{K}-L^{K}\right\|^{2}
\end{aligned}
$$

The same thing whene we use the upper bound $U_{\text {quad }}(x)$ with the equivalent linear form of the objective function $f$ and we obtain: $\left\|f(x)-\widetilde{U}_{\text {quad }}(x): x \in S^{K} \cap\left(D_{f}\right)\right\| \leq\left(\rho(Q+\theta I)+\theta+\frac{1}{8} K\right) h^{2}: h^{2}=\left\|U^{K}-L^{K}\right\|^{2}$ Then, the proof is complete.

\subsubsection{The quadratic approximation error}

Is exhibited by the separation between the capacity $f$ and here lower rough quadratic capacity Lequad over the square shape SK, at that point we have the accompanying recommendation:

Proposition 3.6 : let the function $f: C \subseteq \mathrm{R}^{n}-\rightarrow \mathrm{R}$ where $C \subseteq S^{0} \subseteq$ $\mathrm{R}^{n}$ and $\theta \geq\left|\lambda_{\min }\right|$ for this the matrix $(Q+\theta I)$ be semi-positive, then we have:

$\max _{x \in S^{K} \cap\left(D_{f}\right)}\left\{\left|f(x)-L_{\text {quad }}(x)\right|\right\} \leq\left(\rho(Q+\theta I)+\theta+\frac{1}{2} K\right)\left\|U^{K}-L^{K}\right\|^{2}$
$\max _{x \in S^{K} \cap\left(D_{f}\right)}\left\{\left|f(x)-U_{\text {quad }}(x)\right|\right\} \leq\left(\rho(Q+\theta I)+\theta+\frac{1}{2} K\right)\left\|U^{K}-L^{K}\right\|^{2}$

Proof: By the definition of the function $L_{q u a d}(x)$ as well as the meaning of $\phi_{s} k(x)$, we have:

$$
\begin{aligned}
f(x)-L_{\text {quad }}(x) & =f(x)-L_{S^{K}}(x)+\frac{1}{2} K\left(U^{K}-x\right)\left(x-L^{K}\right. \\
& =\left(x-L^{K}\right)^{T}(Q+\theta I)\left(x-L^{K}\right)+\left(\frac{1}{2} K+\theta\right)\left(U^{K}-x\right)\left(x-L^{K}\right)
\end{aligned}
$$

Then: 


$$
\begin{aligned}
\left\|f(x)-L_{\text {quad }}(x)\right\|_{\infty} & =\max \left\{f(x)-L_{\text {quad }}(x): x \in S^{K} \cap\left(D_{f}\right)\right\} \\
& \leq\left\|\left(x-L^{K}\right)^{T}(Q+\theta I)\left(x-L^{K}\right)\right\|_{\infty}+\left\|\left(\frac{1}{2} K+\theta\right)\left(U^{K}-x\right)\left(x-L^{K}\right)\right\|_{\infty} \\
& \leq\left(\rho(Q+\theta I)+\theta+\frac{1}{2} K\right)\left\|U^{K}-L^{K}\right\|^{2}
\end{aligned}
$$

The same thing whene we use the lower bound $U_{\text {quad }}(x)$ with the equivalent linear form of the objective function $f$ and we obtain:

$\left\|f(x)-U_{\text {quad }}(x)\right\|_{\infty} \leq\left(\rho(Q+\theta I)+\theta+\frac{1}{2} K\right)\left\|U^{K}-L^{K}\right\|^{2}$

So, the proof is complete.

\subsection{The quadratic approximate problem (QAP)}

\subsection{1. costruction of the interpolate problem (IP)}

It's clear that:

$f(x) \geq \max \left\{L_{\text {quad }}(x), U_{\text {quad }}(x): \forall x \in\left(D_{f}\right) \cap S^{K}\right\}=\gamma(x$

This function present the best quadratic lower bound of $f$, similarly, we construct the following interpolate problem by:

$\alpha_{h}=\max x$

$$
\text { b } \quad(\text { LBP })
$$

$x \in\left\{L_{\text {quad }}(x), U_{\text {quad }}(x)\right\}: \forall x \in\left(D_{f}\right) \cap S_{K} \mathrm{~b}$

And the convex quadratic problem define by:

$\min \alpha_{h}$

(AQP) $\forall x \in\left(X_{f}\right) \cap S_{K}$

the question is: what's the relation between the optimal values

$f(x), f\left(x^{*}\right)$ and $L_{q u a d}(x)$ ?

$$
\mathrm{e}
$$$$
\text { e }
$$

We have the following proposition:

Proposition 3.7 : Let the function $f: C \subseteq \mathrm{R}^{n} \rightarrow \rightarrow \mathrm{R}$ and $S^{0} \subseteq \mathrm{R}^{n}$ where $C \subseteq S^{0} \subseteq \mathrm{R}^{n}$, we have:

$$
0 \leq f(\widetilde{x})-f\left(x^{*}\right) \leq\left(\rho(Q+\theta I)+\theta+\frac{1}{2} K\right)\left\|U^{K}-L^{K}\right\|^{2}
$$

$L_{\text {quad }}(\widetilde{x}) \leq f^{*} \leq f(\widetilde{x})$

with $f^{*}=f\left(x^{*}\right)$ is the global optimal value of the original problem

$(N Q P)$ and $x$ be the e optimal solution of (ACQP)

Proof: From the previous proposition, we have:

$f(x)-L_{\text {quad }}(x) \leq\left(\rho(Q+\theta I)+\theta+\frac{1}{2} K\right)\left\|U^{K}-L^{K}\right\|^{2}: x \in S^{K} \cap\left(D_{f}(15)\right.$

And for

$x=\widetilde{x}:$

$$
f(\widetilde{x})-L_{\text {quad }}(\widetilde{x}) \leq\left(\rho(Q+\theta I)+\theta+\frac{1}{2} K\right)\left\|U^{K}-L^{K}\right\|^{2}
$$

Thus:

$f(\widetilde{x})-f^{*}+f^{*}-L_{\text {quad }}(\widetilde{x}) \leq\left(\rho(Q+\theta I)+\theta+\frac{1}{2} K\right)\left\|U^{K}-L^{K}\right\|^{2}$

And:

$f(\widetilde{x})-f^{*} \leq\left(\rho(Q+\theta I)+\theta+\frac{1}{2} K\right)\left\|U^{K}-L^{K}\right\|^{2}+\left(L_{\text {quad }}(\widetilde{x})-f^{*}\right.$

As well as $L_{\text {quad }}(x)-f^{*} \leq 0$, we have: e
$0 \leq f(\widetilde{x})-f^{*} \leq\left(\rho(Q+\theta I)+\theta+\frac{1}{2} K\right)\left\|U^{K}-L^{K}\right\|^{2}$

In the other hand, we have:

$\left\{\begin{array}{c}L_{\text {quad }}(\widetilde{x})-f^{*} \leq 0 \\ f(\widetilde{x})-f^{*} \geq 0\end{array} \Longrightarrow\left(L_{\text {quad }}(\widetilde{x}) \leq f^{*} \leq f(\widetilde{x}\right.\right.$

Then, the proof is complete.

3.5.2. Question: is the solution $x$ present the best lower bound of the globale

optimal solution of (NQP)?

We have the following proposition:

Proposition 3.8 Let take the estimate function noted by:

$E(x):=f(x)-L_{q u a d}(x)$ For all $x \in S^{K} \cap\left(D_{f}\right)$, the foolowing

inequality is satisfied:

$E(x) \geq f(x)-f^{*} \mathrm{e} \quad \mathrm{e}$

\section{Preuve:}

We have:

$f(x)-f^{*}=f(x)-L_{q u a d}(x)+L_{q u a d}(x)-f^{*}$ e e e e

$=E(x)+L_{\text {quad }}(x)-f^{*} \mathrm{e} \quad \mathrm{e}$

And, from the previeus proposition we have:

$L_{\text {quad }}(x) \leq f * \leq f(x)$ e e

So:

$\operatorname{Lquad}(x)-f * \leq 0$ e Then:

$f(x)-f^{*} \leq E(x)$ e $\quad \mathrm{e}$

Lemma: If $E(x)$ is a small value, then $f(x)$ is an acceptable approximative value to e e

the global optimal value $f^{*}=f\left(x^{*}\right)$ over the rectangle $S^{K}$. Similirly, we can find that the point $x$ is the global approximate solution of the global optimal solution $x^{*}$ of the original e problem (NQP) over $S^{K}$.

Preuve: We have:

$f(x)-f^{*} \leq E(x) \mathrm{e} \quad \mathrm{e}$

So, let take that $E(x)$ is a small value we get: e

$f(\widetilde{x})-f^{*} \leq E(\widetilde{x})<<\varepsilon_{\text {with } \varepsilon-\rightarrow 0}$

Then:

$\mathrm{k} f(x)-f * \mathrm{k}<\varepsilon$ e And:

$\lim _{\varepsilon \rightarrow 0}\left\|f(\widetilde{x})-f^{*}\right\|=0$

Immediately, we get that $f(x)$ is an acceptable approximative value to the global optimal e

value $f^{*}=f\left(x^{*}\right)$. Similirly, the point $x$ is a global approximate solution of the global optimal e

solution $x^{*}$ of the original problem (NQP) over $S^{K}$.

In the other hand, the rank of the non convex function $f$ over the new rectangle (subrectangle) $S^{K}$ is small then here rank over the initial rectangle $S^{\circ}$, by this, the value $E(x)$ e will be verry small.

\section{The technical reduction (technical eliminate)}

We get to describe the rectangle partion by the following steps:

$\operatorname{Step}(0)$ :

Let $S^{K}=\left\{x^{k} \in \mathbb{R}^{n}: L_{i}^{K} \leq x_{i}^{k} \leq U_{i}^{K}: i=\overline{1, n}\right\}$ with $x^{k} \in S^{K}$, 
Step(1): We find a partition information point:

$h_{s}=\max \left\{\left(x_{i}-L_{i}^{K}\right)\left(U_{i}^{K}-x_{i}\right): i=\overline{1, n}\right\}$

Step(2): If $h_{s} 6=0$ then we partition the rectangle $S^{K}$ into two subrectangle on edge $\left[L_{s}^{K}, U_{s}^{K}\right]$ by the point $h_{s}$, else, we partition the rectangle $S^{K}$ into two subrectangle on the longest edge $\left[L_{s}^{K}, U_{s}^{K}\right]$ by the middle point $\frac{L^{K}+U^{K}}{2}$ which is yet noted as $h_{s}$.

Step(3): The rest rectangle is yet noted as $S^{K}$.

Now, we describe the rectangle reducing tactics to accelerate the convergence of the proposed global optimization algorithm (ARSR).

\section{Remarks:}

1- All linear constraints of the problem (NQP) are expressed as:

$\sum_{j=1}^{n} a_{i j} x_{j} \leq b_{i}: i=\overline{1, n}$

2- The rectangle $S^{K}$ be also recorded as constraint to be added to the problem (NQP).

3- The minimum and the maximum of each function:

$$
\left\{\begin{array}{c}
\psi\left(x_{i}\right)=a_{i s} x_{s}: i=\overline{1, n} \\
x_{i} \in\left[L_{s}^{K}, U_{s}^{K}\right]
\end{array}\right.
$$

Are obtaind at the extremes points of the same interval.

\section{Linearity Based Range Reduction Algorithm:}

This algorithm is given to reduce and delete the rectangle $S^{K}$.

\section{program (LBRRA)}

Let $I_{k}^{\prime}:=\{1,2,3, \ldots, n\}$ the set of the index, $P_{k}:=P$ for $1 \leq i$ $\leq n$ do

$$
r U_{i}:=\sum_{j=1} \max \left\{a_{i j} L_{j}^{k}, a_{i j} U_{j}^{k}\right\}
$$

compute

com-$$
r L_{i}:=\sum_{j=1} \min \left\{a_{i j} L_{j}^{k}, a_{i j} U_{j}^{k}\right\}
$$

if $r L_{i}>b_{i}$ then

stop. the problem (NQP) is infeasible over $S^{K}$ (there are no solution of (NQP) over $S^{K}$,because, $S^{K}$ is deleted From the subrectangle set produced through partitioning of the rectangle $S^{\circ}$ ) else if $r U_{i}<b_{i}$ then the constraint is redundant.

$I_{k}^{\prime}:=I_{k}^{\prime}-\{i\}$

$P_{k}:=P_{k}-\left\{x \in \mathbb{R}^{n}:\left(a_{i}\right)^{T} x \leq b_{i}\right\}$

else

for $1 \leq j \leq n$ do if $a_{i j}>0$ then

$U_{j}^{k}:=\min \left\{U_{j}^{k}, \frac{b_{i}-r L_{i}+\min \left\{a_{i j} L_{j}^{k}, a_{i j} U_{j}^{k}\right\}}{a_{i j}}\right\}$

else

$L_{j}^{k}:=\max \left\{L_{j}^{k}, \frac{b_{i}-r U_{i}+\max \left\{a_{i j} L_{j}^{k}, a_{i j} U_{j}^{k}\right\}}{a_{i j}}\right\}$

end if

anddo end if

end if enddo end program

\section{Algorithm (ARSR): Branch and Bound}

\subsection{Algorithm (ARSR): Branch and Bound program (ARSR)}

Initialization: Determine the initial rectangle $S^{0}$ where $\left(\chi_{f}\right) \subset S^{0}$ and suppose that:

$Q L B P_{S} 0:=S^{0} \cap\left(\chi_{f}\right)$

iteration $k$ :

if $Q L B P_{S} 06=\varphi$ then

solve the quadratic problem (LBP) when $k=0$

Let $x^{0}$ be an optimal solution of (LBP) and $\alpha\left(S^{0}\right)$ be the optimal value acompaned to $x^{0} H:=\left\{S^{0}\right\}$ (the set of the subrectangle of the initial rectangle $\left.S^{0}\right) \alpha_{0}:=\min \left\{\alpha\left(S^{0}\right)\right\}, \beta_{0}:=f\left(x^{0}\right)$ (the upper bound of $\left.f\left(x^{*}\right)\right) k:=0$ while Stop=false do if $\alpha_{k}=\beta_{k}$ then

Stop=true $\left(x^{k}\right.$ is a global optimal solution of the problem (NQP)) else we subdivise the rectangle $S^{k}$ into two sub-rectangle $\left\{S_{j}^{k}: j=\right.$ $1,2\}$ by the proposed algorithm. for $j=1,2$ do applied the Linearity Based Range Reduction Algorithm over the two sub-rectangle

$\{S j k\}$ the obtained set is yet noted as the rectangle $S_{j}^{k}$ if $S_{j}^{k} 6=\varphi$ then

$(Q L B P)$ sjk $:=\left\{x \in \mathrm{R}^{n}: x \in S_{j}^{k} \cap\left(\chi_{f}\right)\right\}$, solve the quadratic problem $(Q L B P)$ when $S^{k}:=S_{j}^{k}$ let $x^{k j}$ be the optimal solution and $\alpha\left(S_{j}^{k}\right)$ be the optimal value $H:=H \cup\left\{S_{j}^{k}\right\}$

$\beta_{k+1}:=\min \left\{f\left(x^{k}\right), f\left(x^{k j}\right)\right\} x^{k}:=\arg \min \beta_{k+1}$ end if

end for

$H:=H-\left\{S^{k}\right\}$

$\alpha_{k+1}:=\min _{S \in H}\{\alpha(S)\}$; choose an rectangle $S^{k+1} \in H$ such that $\alpha_{k+1}=$ $\alpha\left(S^{k+1}\right)$

$k \leftarrow k+1$

end if

end do end if end program

\section{The convergence of the Algorithm (ARSR)}

In this area, we examine the union of the proposed calculation (ARSR) and we give a straightforward examination between the direct surmised and the quadratic one. In the other hand, we give some guide to expline the proposed calculation.

\subsection{The convergence of the proposed algorithm}

The proposed calculation in area 5 is not the same as the one in ref [3] in lower-jumping (quadratic estimate), and added to the square shape decreasing methodology. We will demonstrate that the proposed calculation be united.

Hypothesis 6.1 : On the off chance that the proposed calculation ends in limited advances, at that point a worldwide ideal arrangement of the issue (NQP) is gotten when the calculation ends.

Confirmation: Let the outcome out coming when the calculation end be $\mathrm{xk}$, at that point, quickly we have $\mathrm{ax}=\mathrm{Bk}$ while ending at the- $k$-step, so $x^{k}$ is a global optimal solution of the problem(NQP).

Theorem 6.2 If the algorithm generates an infinite sequence $\left\{x^{k}\right\}$ global optimal solution of the problem $(N Q P)($ i.e: the global optimal solution is not unique). 
Proof: Let $x^{*}$ be an accumulation point of the sequence $\left\{x^{k}\right\}_{k \in \mathbb{N}^{*} \text { and let }}\left\{x_{p}^{k}\right\}_{k \in \mathbb{N}^{*}, p \in \mathbb{N}^{*} \text { be a subsequence of the }}$ sequence $\left\{x^{k}\right\}_{k \in \mathbb{N}^{*} \text { converging to } x^{*} \text {. obviously in the proposed }}$ algorithm, the lower sequence $\left\{a_{k}\right\}_{k \in \mathrm{N}^{*}}$ is mono- increase and the upper sequence $\left\{B_{k}\right\}_{k \in N^{*}}$ is mono-

decrease, and we have:

$\alpha k=\operatorname{lquad}(x k), B k=f(x k)$

We can right:

$\alpha_{k}=l_{\text {quad }}\left(x^{k}\right) \leq \min _{x \in S_{k}} f(x) \leq B_{k}=f\left(x^{k}\right.$

So both $\left\{x_{k}\right\}_{k \in N^{*}}$ and $\left\{B_{k}\right\}_{k \in N^{*}}$ are convergent and:

$\lim B_{k}=\lim B_{k q}=\lim f\left(x^{k}\right)=\lim f\left(x^{k q}\right)=f\left(x^{*}\right)$

$k \rightarrow \infty \quad q \rightarrow \infty \quad k \rightarrow \infty \quad q \rightarrow \infty$

Without loss of generality, we assume that $x^{k q}$ is the solution of the problem $(Q L B P)$ on

$S_{k q}$ which satisfies $S_{k q+1} \subset S_{k q}, q \geq 1$, by the proprieties of the proposed rectangle partition which is exhaust, i.e:

$\lim S_{k q}=x^{*} q \rightarrow \infty$

We have:

$0 \leq B_{k_{q}}-\alpha_{k_{q}}=f\left(x_{q}^{k}\right)-l_{\text {quad }}\left(x_{q}^{k}\right) \leq\left(\rho(Q+\theta I)+\theta+\frac{1}{2} K\right)\left\|U_{q}^{K}-L_{q}^{K}\right\|^{2}$

Then:

$\lim _{q \rightarrow 0}\left(f\left(x_{q}^{k}\right)-l_{\text {quad }}\left(x_{q}^{k}\right)\right)=\lim _{q \rightarrow 0}\left(B_{k_{q}}-\alpha_{k_{q}}=0\right.$

Thus, we have:

$\lim _{q \rightarrow 0}\left(B_{k_{q}}-\alpha_{k_{q}}\right)=\lim _{q \rightarrow 0}\left(\alpha_{k_{q}}-B_{k_{q}}-\left(B_{k_{q}}-\alpha_{k_{q}}\right)\right)=0$

So:

$\lim _{k \rightarrow 0} \alpha_{k}=\lim _{q \rightarrow 0} \alpha_{k_{q}}=\lim _{q \rightarrow 0}\left(B_{k_{q}}-\left(B_{k_{q}}-\alpha_{k_{q}}\right)\right)=\lim _{q \rightarrow 0} B_{k_{q}}$

And, from (I) we have:

$\lim _{k \rightarrow 0} \alpha_{k}=\lim _{q \rightarrow 0} B_{k_{q}}=\lim _{q \rightarrow \infty} f\left(x^{k_{q}}\right)=f\left(x^{*}\right)$

Therefore, the point $x^{*}$ is an global optimal soluion of the problem (NQP).

\subsection{The type and rank of convergence}

The proposed calculation unite to the rough arrangement of the ideal worldwide arrangement of the first issue (NQP) with a quadratic vitesse over SK.

In this strategy, the position of the non curved capacity f over the square shape SK will be lower then his position over the underlying one $\mathrm{S}$, along these lines immetiately give that the esteem $\mathrm{E}(\mathrm{x})$ is e verry little. By this outcome, the arrangement point $\mathrm{x}$ is a globale inexact answer for the worldwide e

ideal arrangement $\mathrm{x} *$ over SK.

To quicken the assembly of the proposed calculation we utilized the specialized of dividing and decreasing where in each progression we take out a square shape and a direct limitation, and this give us a square shape littler then the intial one and we signified it by SK.

\section{7. comparison between "Branch and Bound" and "Method (DCT)"}

\subsection{Method (DCT)}

We should include the ordinariness condition characterize by the decision of the parametre $\mu>0$ so as to garante the presence of the worldwide ideal arrangement, this condition is given by:

\subsubsection{Introduction}

Let take the non convex quadratic optimization problem given by:

$\left\{\begin{array}{c}\min f(x)=\frac{1}{2} x^{T} Q x-d^{T} x \\ A x \leq b ; \quad x \geq 0\end{array}\right.$

where $\quad A \in \mathrm{R}^{n \times m}$ arbitrary matrix $b, x$ vertex of $\mathrm{R}^{n}$

The fondamental idea of this method is in the chose of the operator:

$\Lambda(x): \mathrm{R}^{n} \rightarrow \mathrm{R}^{m}$

By this the objective function $f$ be write as the following canonical form:

$f(x)=\Phi(x, \Lambda(x))$

Define over the set $\mathrm{R}^{n} \times \mathrm{R}^{m}$ to $\mathrm{R}$ in the condition that the function be canonic at every unone (point) $x$ and $y$.

We need the following definitions:

Remark: The canonical function $\Phi(x, \Lambda(x))$ can represent by:

$\overline{\Phi(x}, \Lambda(x))=\bar{W}(y)-F(y): y \in \mathrm{R}^{m}$

This function is defind over $\mathrm{R}^{m} \times \mathrm{R}^{n}$ to $\mathrm{R}$

In the other hand, we use the dual $\Lambda$-canonical transformation to calculate the conjugate

function of $F(y)$ given by:

$\bar{F}^{\Lambda}\left(y^{*}\right)=\left\{(\Lambda(x))^{T} y^{*}-\bar{F}(x): \Lambda_{t}^{T}(x) y^{*}-D \bar{F}(x)=0\right\}$ with:

$\Lambda_{t}^{T}(x)=D \Lambda(x)$

By the use of this notions, we can construct the associate dual function of $f$ by:

$f^{d}\left(y^{*}\right)=\bar{F}^{\Lambda}\left(y^{*}\right)-\bar{W}^{*}\left(y^{*}\right)$

\subsubsection{Method (DCT) for the non convex quadratic problems}

At that point, we connected the strategy (DCT) over the partner parametric issue (PQP) in the spot of the non covex quadratic issue (NQP) like folow:

$|x|^{2} \leq 2 \mu$

Then, we have:

$\left\{\begin{array}{c}\min f(x)=\frac{1}{2} x^{T} Q x-d^{T} x \\ A x \leq b ; \quad x \geq 0 ;|x|^{2} \leq 2 \mu\end{array}\right.$

$(\mathrm{PQP})$

We can transform the problem $(\mathrm{PQP})$ as:

$$
\begin{aligned}
& \int \min f(x)=\frac{1}{2} x^{T} Q x-d^{T} x \\
& A x \leq b ; \frac{1}{2}|x|^{2} \leq \mu \\
& A \quad(n+1) \times n \quad b \quad n+1 \\
& A=(\quad) \in \mathrm{R} \quad \text { and } b=() \in \mathrm{R} \\
& -1-1-1 . .-1 \quad 0
\end{aligned}
$$

At that point, we connected the strategy (DCT) over the partner parametric issue (PQP) in the spot of the non covex quadratic issue (NQP) like folow: 
Step(1): The form of the operator $\Lambda(x)$ :

For this type of problem the canonical geometric operator:

$\Lambda(x): \mathrm{R}^{n} \rightarrow \mathrm{R}^{m} \times \mathrm{R}$

Is define by:

$y=\Lambda(x)=\left(A x, \frac{1}{2}|x|^{2}\right)=(\varepsilon, \rho) \in \mathbb{R}^{m} \times \mathbb{R}$

And, it's presented as an Vertex-Value application.

By this, the realisable domain of (PQP) will be define by:

$D_{P Q P}=\left\{y=(\varepsilon, \rho) \in \mathrm{R}^{m} \times \mathrm{R}: \varepsilon \leq b, \rho \leq \mu\right\}$

Step(2): The structure of the function $W(y)$ :

In this case, the function $W(y) \quad \bar{W} \quad: \quad \mathbb{R}^{n} \rightarrow \mathbb{R}$ is given by the Indicative function of the realisable domain $D_{P Q P}$ like folows:

$$
\mapsto \bar{W}()=\{
$$

$\in D_{P Q P}$ $\begin{array}{ll}y & y \\ +\infty & \text { else }\end{array}$

Then, it's clear that the function $W(y)$ is always convex from the propriety of the indicative

function. In the other hand, the function $W(y)$ is proper and slower continuous over the set $D_{P Q P}$.

By this we have:

$\left.*^{*}\right)$ :

$\operatorname{Step}(3)$ : The structure of the function $W(y$

$$
\begin{aligned}
& \bar{W}^{*}\left(y^{*}\right)=\sup _{y \in D_{P Q P}}\left\{\left\langle y, y^{*}\right\rangle-\bar{W}(y)\right\} \\
& =\sup _{\varepsilon \leq b} \sup _{\rho \leq \mu}\left\{(\varepsilon, \rho)^{T}\left(\varepsilon^{*}, \rho^{*}\right)-\bar{W}(y): y \in D_{P Q}\right. \\
& =\sup \sup \left\{\varepsilon^{T} \varepsilon^{*}+\rho^{T} \rho^{*}: y \in D_{P Q P}\right\} \\
& \text { if } \quad \varepsilon^{*} \geq 0, \rho^{*} \geq 0 \text { else } \\
& =\left\{\begin{array}{c}
\varepsilon^{T} \varepsilon^{*}+\rho^{T} \rho^{*} \\
+\infty
\end{array}\right.
\end{aligned}
$$

Step(4): The structure of the function $F(y$

The function $F(y)$ is a linear function, and we have:

$\overline{f(x)}=\Phi(x, \Lambda \overline{(x)})=W(y)-F(y): y \in \mathrm{R}^{m} \times \mathrm{R}$

Then, we get:

$\overline{f(x)}-W(y)=-\overline{F(y)}: y \in \mathrm{R}^{m} \times \mathrm{R}$

And for $y \in D_{P Q P}$ we have:

$\overline{-f}(x)=F(y)$

Immediately, the $\Lambda$-canonical conjugate of the function $F(y)$ is define by:

$$
\begin{aligned}
\bar{F}^{\Lambda}\left(y^{*}\right) & =\sup _{y \in D_{P Q P}}\left\{y^{T} y^{*}-\bar{F}(y): \Lambda_{t}^{T}(x) y^{*}-D \bar{F}(x)=0: x \in D_{P Q P}\right\} \\
& =\sup _{y \in D_{P Q P}}\left\{(\Lambda(x))^{T} y^{*}-\bar{F}(y): \Lambda_{t}^{T}(x) y^{*}-D \bar{F}(x)=0: x \in D_{P Q P}\right\}
\end{aligned}
$$

And, from the first step we have:

$y=\Lambda(x)=\left(A x, \frac{1}{2}|x|^{2}\right)=(\varepsilon, \rho) \in \mathbb{R}^{m} \times \mathbb{R}$

Thus:

$$
\begin{aligned}
& \bar{F}^{\Lambda}\left(y^{*}\right)=\sup _{y \in D_{P Q P}}\left\{(\Lambda(x))^{T} y^{*}-\bar{F}(\Lambda(x)): \Lambda_{t}^{T}(x) y^{*}-D \bar{F}(x)=0: x \in D_{P Q P}\right\} \\
& =\sup _{y \in D_{P Q P}}\left\{\frac{1}{2} x^{T}\left(Q+\rho^{*} I\right) x-\left(d-A^{T} \varepsilon^{*}\right)^{T} x\right\}: x \in D_{P Q P} \\
& =\frac{-1}{2}\left(d-A^{T} \varepsilon^{*}\right)^{T}\left(Q+\rho^{*} I\right)^{-1}\left(d-A^{T} \varepsilon^{*}\right) \text { with } x \\
& =\left(Q+\rho^{*} I\right)^{-1}\left(d-A^{T} \varepsilon^{*}\right)
\end{aligned}
$$

Step(5): The structure of the dual canonical function $f^{d}\left(y^{*}\right)$ :

Finally, and from the forth step, we define the dual canonical function by:

$$
\begin{aligned}
f^{d}\left(y^{*}\right) & =\bar{F}^{\Lambda}\left(y^{*}\right)-\bar{W}^{*}\left(y^{*}\right) \\
& =\frac{-1}{2}\left(d-A^{T} \varepsilon^{*}\right)^{T}\left(Q+\rho^{*} I\right)^{-1}\left(d-A^{T} \varepsilon^{*}\right)-\varepsilon^{T} \varepsilon^{*}-\rho^{T} \rho^{*}:\left(\varepsilon^{*}, \rho^{*}\right) \in \mathbb{R}^{m} \times \mathbb{R}
\end{aligned}
$$

Then, the parametric dual problem is given by:

$$
\begin{gathered}
\max _{*} \geq 0, \rho^{*} \geq 0, \operatorname{det}\left(Q *, \rho^{*}\right) \\
\left(\rho^{*} I\right) 6=0 \quad(\mathrm{CPD})
\end{gathered}
$$

$\varepsilon$ We can find an equivalence between the primal problem and the dual one, that's given by the following theorem:

Theorem 7.1 [1]: If $\overline{y^{*}}=\left(\overline{\varepsilon^{*}}, \overline{\rho^{*}}\right)$ be a $($ K.K.T) point of the parametric dual problem $(C P D)$ then the vertex $x=\left(Q+\rho^{*} I\right)^{-1}\left(d-A^{T} \varepsilon^{*}\right) \mathrm{e}$

is a (K.K.T) point of the parametric primal problem $(P Q P)$, and we have:

$f^{l}\left(y^{*}\right)=f(x) \mathrm{e}$

Remark: Let take $i d$ be the number of the negative distincts eigenvalues of the matrix $Q$ then, the quadratic problem be non convex if $i d>0$.

\subsection{Convergence Theorem of the method (DCT)}

We can assume the inquiry "what's the connection between the ideal arrangements of the parametric issue (PQP), the base issue $(\mathrm{NQP})$ and the parametric double issue (CPD)??

To give the appropriate response we have this hypothesis:

Theorem 7.2 [1]:Let $Q$ a matrix with the index id $>0$ and $\left\{\lambda_{i}\right\}_{i=1}, p: p \leq n$ a distincts eigenvalues in the order: $\lambda 1<\lambda 2<\ldots<\lambda i d<0 \leq \lambda i d+1<\lambda i d+2<\ldots<\lambda p$ and let $\left(\overline{\varepsilon^{*}}, \overline{\rho^{*}}\right)$ be a K.K.T point of the parametric dual problem $(C P D)$, and let:

$x=\left(Q+\rho^{*} I\right)^{-1}\left(d-A^{T} \varepsilon^{*}\right) \mathrm{e}$

be a K.K.T point of the prametric primal problem $(P Q P)$, then we have:

1 If $\overline{\rho_{i}^{*}}>-\lambda_{1}>0$ then, the vertex $\left(\varepsilon^{*}, \rho^{*}\right)$ is a maximum of $f^{l}\left(y^{*}\right)$ over $D_{P Q P^{+}} \quad$ if and only if the vertex $x$ e is a minimum of $f(x)$ over $D_{P Q P} P^{s} \quad, \quad$ and we write:

$f\left(\widetilde{x}_{i}\right)=\min _{x \in \underline{D_{P Q P}^{s}}} f(x)=\max _{\left(\varepsilon^{*}, \rho^{*}\right) \in D_{P Q P}^{+}} f^{d}\left(\varepsilon^{*}, \rho^{*}\right)=f^{d}\left(\overline{\varepsilon^{*}}, \overline{\rho^{*}}\right)$

2 If $0 \leq \rho^{*}{ }_{i}<-\lambda_{\text {id }}$ then, the vertex $\left(\overline{\varepsilon^{*}}, \overline{\rho^{*}}\right)$ is a maximum of $f^{d}\left(y^{*}\right)$ over $D_{P Q P}$ if and only if the vertex $x$ is a global maximum of $f(x)$ over $D_{P Q P}$, and we write: $\mathrm{e}$

$f\left(\widetilde{x}_{i}\right)=\max _{x \in \underline{D_{P Q P}}} f(x)=\max _{\left(\varepsilon^{*}, \rho^{*}\right) \in D_{P Q P}^{-}} f^{d}\left(\varepsilon^{*}, \rho^{*}\right)=f^{d}\left(\overline{\varepsilon^{*}}, \overline{\rho^{*}}\right)$

3 If $0<\rho^{*}{ }_{i}<-\lambda_{\text {id }}$ then, the vertex $\left(\overline{\varepsilon^{*}}, \overline{\rho^{*}}\right)$ is a minimum of $f^{d}\left(y^{*}\right)$ over $D_{P Q P} P^{i}$ if and only if the vertex $x$ is a global minimum of $f(x)$ over $D_{P Q P}$, and we write: $\mathrm{e}$ 
$f\left(\widetilde{x_{i}}\right)=\min _{x \in D_{P Q P}} f(x)=\min _{\left(\varepsilon^{*}, \rho^{*}\right) \in D_{P Q P}^{i}} f^{d}\left(\varepsilon^{*}, \rho^{*}\right)=f^{d}\left(\overline{\varepsilon^{*}}, \overline{\rho^{*}}\right)$

\subsection{Examples}

\subsubsection{Example1}

Let the non convex quadratic function define by:

$f(x)=\left(x_{1}+1\right)^{2}+\left(x_{2}+1\right)^{2}-\frac{5}{2}\left(x_{1}+x_{2}\right)-3\left(x_{1}^{2}+x_{2}^{2}\right)-{ }_{2}$

So, we have:

$$
\begin{aligned}
& L_{\text {quad }}(x)=\left(x_{1}^{2}+x_{2}^{2}\right)+\frac{3}{2}\left(x_{1}+x_{2}\right)-2 \\
& \widetilde{L}_{\text {quad }}(x)=\frac{1}{2}\left(x_{1}+x_{2}\right)-2-\frac{1}{8}(3)
\end{aligned}
$$

Figure 1: Figure 1

With:

$$
\begin{array}{lll}
f(x) \quad: & \text { broun whith black } \\
\mathscr{L}_{\text {quad }}(x) \quad: & \text { red whith yellow } \\
L_{\text {quad }}(x) & : \quad \text { darkgray whith navy }
\end{array}
$$

The graphic representation of the non convex quadratic function $f$, the linear approximate function and the convex quadratic lower bound function over the rectangle $[-1,0] \subseteq \mathrm{R}^{n}$

Plainly the raised quadraic inexact capacity is between the target work and the straight rough one of a similar capacity over he square shape $S^{0}=[-1,0] \subseteq \mathrm{R}^{n}$

\subsubsection{Example2}

Let take the following quadratic programming problem:

$\left\{\begin{array}{c}\min f(x)=\frac{1}{2} a x^{2}-d x \\ |x| \leq r\end{array}\right.$

So,if $a \geq 0$ then, the problem be convex and this case is simple to resolve, however, if $a<0$.

Let $a=-6, d=4$ and $r=1.5$, then:

$(\min f(x)=-3 \times 2-4 x$

$|x| \leq 1.5$

Figure 2: Figure 2

This function accept one and only extrema in the point $x=-\frac{2}{3}$ with the associate value

$f(x)=\frac{4}{3}$

And, by the use of the dual canonical transformation, we can define the associate dual forme of $f$ by:

$$
\begin{aligned}
f^{d}\left(\rho^{*}\right) & =\frac{-1}{2} d\left(a+\rho^{*}\right)^{-1} d-\mu \rho^{*} \\
& =\frac{-1}{2}(16)\left(-6+\rho^{*}\right)^{-1}-\frac{1}{2}(1.5)^{2} \rho^{*} \\
& =-\left(1.125 \rho^{*}+\left(\frac{8}{\rho^{*}-6}\right)\right)
\end{aligned}
$$

In the other part, the dual canonical problem is given by:

$$
\left\{\begin{array}{c}
\max f^{d}\left(\rho^{*}\right)=-\left(1.125 \rho^{*}+\left(\frac{8}{\rho^{*}-6}\right)\right) \\
\rho^{*} \geq 6
\end{array}\right.
$$

$f(x)$ : black $f^{d}\left(\rho^{*}\right)$ : broun

Candidate(s) for

$\{-0.75,-12.75\}$, at $\left\{\left[\rho_{1}^{*}=3.3333\right],\left[\rho_{2}^{*}=8.6667\right]\right\}$

So, we have the following results:

\begin{tabular}{|l|l|l|}
\hline functions & extremas & candidates for extremas \\
\hline primal & $-0,6666$ & 1,3333 \\
\hline \multirow{2}{*}{ dual } & 3,3333 & $-0,7500$ \\
& 8,6667 & $-12,7500$ \\
\hline
\end{tabular}

With:

$\widetilde{x_{1}}=\left(a+\overline{\rho_{1}^{*}}\right)^{-1} d=-1,4998$

$\widetilde{x_{2}}=\left(a+\overline{\rho_{2}^{*}}\right)^{-1} d=1,5000$

Immediately, we have this table:

\begin{tabular}{|l|l|l|l|}
\hline \hline $\begin{array}{l}\text { dual } \\
\text { extremas } \\
\rho^{*}{ }_{i}\end{array}$ & $\begin{array}{l}\text { Primal } \\
\text { solutions } \\
x_{i} \mathrm{e}\end{array}$ & values $f\left(x_{i}\right) \mathrm{e}$ & dual values \\
\hline 3,3333 & $-1,4998$ & $-0,7490$ & $-0,7500$ \\
\hline 8,6667 & 1,5000 & $-12,7500$ & $-12,7500$ \\
\hline
\end{tabular}

In the other hand, we find the following results:

$\overline{\rho_{1}^{*}}=3,3333<-a=6$

With:

$f\left(\widetilde{x_{1}}\right)=\min _{x \in D_{P Q P}} f(x)=\min _{\left(\rho^{*}\right) \in D_{P Q P}^{i}} f^{d}\left(\rho^{*}\right)=f^{d}\left(\overline{\rho_{1}^{*}}\right)=-0,75$

And:

$\overline{\rho_{2}^{*}}=8,6667>-a=6$

With:

$f\left(\widetilde{x_{2}}\right)=\min _{x \in D_{P Q P}^{s}} f(x)=\max _{\left(\rho^{*}\right) \in D_{P Q P}^{+}} f^{d}\left(\rho^{*}\right)=f^{d}\left(\overline{\rho_{2}^{*}}\right)=-12,75$

So, by the use of the "Branch and Bound method" the convex approximate quadratic form of $f$ is given by:

$L_{\text {quad }}(x)=\frac{1}{2} x^{2}+\frac{7}{4} x$

And the convex approximate quadratic problem associate to the non convex one is given by:

$\left\{\begin{array}{c}\min L_{\text {quad }}(x)=\frac{1}{2} x^{2}+\frac{7}{4} x \\ x \in\left[0, \frac{1}{2}\right]\end{array}\right.$

Where we appleid the reducting and eleminate techenic over the initial ractangle

$S^{\circ}:=\left[\frac{-1}{2}, \frac{1}{2}\right]$

And we find that the rest rectangle is:

$S^{1}:=\left[0, \frac{1}{2}\right]$

So, we have this graph:

$f(x) \quad$ : black

$f^{l}\left(\rho^{*}\right) \quad$ : broun

$(-12,75: \quad$ lightred

) and

$(-0,75)$

$L_{\text {quad }}(x)$ lightblue

Figure 3: Figure 3 
The graphic representation of the primal function $f$, the associate dual function $f^{l}$ and the convex quadratic approximate function $L_{\text {quad }}(x)$

So, over the recangle $S^{1}:=\left[0, \frac{1}{2}\right]_{\text {we find that: }}$

- $\left(\frac{1}{2}\right)$

2 ) is the minimum point of the function $f$ and it is the maximum point of the convex quadratic function $L_{\text {quad }}$ and the minimum point of the associate dual function $f^{l}$ over

$$
\text { the rectangle } S^{1}:=\left[0, \frac{1}{2}\right] .
$$

- (0) is the maximum point of $f$ and the minimum point of the convex quadratic function

$L_{\text {quad }}$ and: $f(0)=L_{\text {quad }}(0)<f^{l}(0)$

\section{8. conclusion}

In this paper we present another square shape Branch and Headed methodology for taking care of non curved quadratic programming issues were we propose another lower rough raised quadratic elements of the target quadratic capacity fover a $n$-rectangle.

This lower surmised is given to decide a lower bound of the worldwide ideal estimation of the first issue (NQP) over every square shape.

To quicken the combination of the proposed calculation we utilized a basic two-segment and decreasing method over the subrectangles SK in the $\mathrm{k}$ - step [3].

In the other hand, we presente an other worldwide technique to determine the issue (NQP), this strategy is "the double standard change (DCT)". This strategy change a non raised quadratic issue to an Algebric framework.

It's dependably unite to the worldwide ideal arrangement over the feasible space wich is a minimal arrangement of $\mathrm{Rn}$.

Figure 4: Figure 4

The new calculation $B \& B$ where we utilized the curved quadratic estimate of the non arched quadratic capacity $\mathrm{f}$ over a rectan${ }_{\text {gle }} S^{K}=\left[L^{K}, U^{K}\right] \subseteq \mathbb{R}^{n}$ with $\theta \geq\left|\lambda_{\min }\right|$ and it is not impty, convex, close, and bounded (compact) of $\mathrm{R}^{n}$ is best at that point the strategy (DCT) over the relative Intrior of the feasible space of the capacity wich we streamlined.

We can utilize the Branch and Bound technique (Partition and assessment) where we compose the capacity f like a (DC) structure (reverence of tow arched capacities) and we estimated the curved part by a raised quadratic capacity by the utilization of the lower bound or the upper bound of the feasible square shape SK wich have a verry little position and it's considred as a confianced locale, and by this we guaranteed the existance of the ideal worldwide arrangement of the first issue (NQP).

In the other hand, the "Branch and Bound strategy" acquire the rough ideal arrangement of the ideal worldwide arrangement of the first issue (NPQ) with a quadratic vitesse of union over the feasible set SK, yet the (DCT) technique locate the ideal worldwide arrangement over the Spher of this feasible set SK.

\section{References}

[1] Gasmi.B, contriution 'a l' etude des M'ethodes de $r$ 'esoluion des probl'emes d'optimiations quadratiques. th`ese de magister (2007).

[2] G.A. Anastassiou and O. Duman, Intelligent mathematics II, Applied mathematics and approximation theory. Vol 441, DOI 10.10070/978-3-319-30322-2 7, (2016) pp 105-117 .

[3] Hongwei Jiao, a Branch and Bound algorithm for globally solving a class of non convex programming problems, Non linear analysis 70 (2009) pp 1113-1123.

[4] Panos. M. Pardalos, Global optimization algorithms for linearly constrained indefinite quadratic problems, Computers math app lic. Vol. 21, NO 6/7, (1991) pp 87-97.

[5] Reiner Horst. Panous. M. Pardalos and Ngugen V. Thoai, Introduction to global optimization. Kluwer academic publishers. Dord Echt/ Boston/ London. Volume 3.(1995).

[6] Xue Honggang, Xu Chengxian, A Branch and Bound algorithm for solving a class of DC-Programming. Applied mathematics and computation $165,(2005)$ pp 291-302 .

[7] Yuelin Gao, Honggang Xue, Peiping Shen, A new rectangle Branch and Bound reduce approch for solving non convex quadratique programming problems, Applied mathemetics and computation 168 (2005), pp 1409-1418. 